\title{
Conditioned suppression in a virtual environment ${ }^{\text {is }}$
}

\author{
W. James Greville ${ }^{\mathrm{a}, \mathrm{b}}$, Philip M. Newton ${ }^{\mathrm{b}}$, Bryan Roche ${ }^{\mathrm{c}}$, Simon Dymond ${ }^{\mathrm{a}, *}$ \\ ${ }^{a}$ Department of Psychology, Swansea University, Singleton Park, Swansea SA2 8PP, United Kingdom \\ ${ }^{\mathrm{b}}$ College of Medicine, Swansea University, Singleton Park, Swansea SA2 8PP, United Kingdom \\ ${ }^{\mathrm{c}}$ Department of Psychology, National University of Ireland, Maynooth, Co. Kildare, Ireland
}

\section{A R T I C L E I N F O}

\section{Article history:}

\section{Keywords:}

Conditioned suppression

Conditioning

Virtual environment

First person perspective video game Anxiety

\begin{abstract}
A B S T R A C T
Virtual environments (VEs) provide an inexpensive way of conducting ecologically valid psychological research. The present study used a VE to demonstrate conditioned suppression, a behavioral model of anxiety, in a first-person perspective video game. During operant training, participants learned to shoot crates to find gold bars and thus score points in the game. Next, during Pavlovian conditioning, a colored light (i.e., conditioned stimulus: CS+) was followed by a white noise unconditioned stimulus (US) while a different colored light ( $\left.C S_{-}\right)$was not paired with the US. Probe trials in a final testing phase were then used to assess suppression. We found significant suppression of accurate responding (shots hitting the designated targets) during the presence of the $\mathrm{CS}+$ relative to the $\mathrm{CS}-$, both in terms of total hits and hits as a proportion of total shots. Importantly, this effect emerged despite the overall level of operant responding being undiminished during the CS+. Our findings are consistent with related studies examining human behavior in real environments, and demonstrate the potential of VEs in combination with a modestly aversive CS to allow a detailed behavioral profile of anxiety to emerge.
\end{abstract}

(c) 2012 Elsevier Ltd. All rights reserved.

\section{Introduction}

Virtual environments (VEs) are an increasingly popular medium for investigating a range of psychological phenomena (Baumann \& Sayette, 2006; Difede \& Hoffman, 2002). VEs provide participants with a more immersive experience than traditional computerbased experiments, thus promoting more "natural" behavior and producing ecologically valid results (Blascovich et al., 2002; Guitton, 2012; Lomanowska \& Guitton, 2012). Indeed, a growing body of literature demonstrates that human behavior in VEs mirrors that in real environments (Kozlov \& Johansen, 2010; Slater et al., 2006). At the same time, the components and properties of VEs can be fully specified by the experimenter, which allows for the delivery of stimuli to be precisely controlled, as well as allowing participants to be exposed to situations that might be impractical or unethical to present in the real world. Thus, while psychological studies often face a compromise between experimental control and ecological validity, VEs allow for both.

Fully immersive VEs such as a "cave" (see, e.g., Huff, Zielinski, Fecteau, Brady, \& LaBar, 2010) can be difficult and costly to create and maintain. An alternative approach is to use a first-person perspective video game. In such games, the VE is displayed on a com-

\footnotetext{
This research was supported by a grant from the BIAL Foundation (106/10) Please contact the corresponding author for further technical details about the video game employed in the present study.

* Corresponding author. Tel.: +44 1792 295602; fax: +44 1792295679.

E-mail address: s.o.dymond@swansea.ac.uk (S. Dymond).
}

puter monitor with a joystick or joypad used to control movement throughout the game. Video game VEs have the advantages of being considerably more affordable and therefore more accessible than the immersive form, and due to the widespread use of video games as a recreational activity, participants may adapt to the task demands far more readily.

Here, we report preliminary findings from a task utilizing a first-person perspective video game VE designed to demonstrate conditioned suppression, a widely studied associative learning model of anxiety (Di Giusto, Di Giusto, \& King, 1974; Estes \& Skinner, 1941). First, we describe the steps taken to implement a system for the recording of responses within a video game that makes it suitable for use as a psychology software tool, and the application of this system to develop a novel conditioned suppression paradigm. Second, we describe findings from an experiment making use of this paradigm, and finally, we discuss the implications of the current approach for empirical investigations of anxiety and other clinical disorders.

\subsection{Experimental psychopathology of fear: The conditioned suppression paradigm}

Within experimental psychopathology, fear conditioning is a widely employed laboratory paradigm used to understand the acquisition and expression of adaptive fear (Beckers, Krypotos, Boddez, Effting, \& Kindt, in press; Mineka \& Oehlberg, 2008). Decades of research has shown that when a previously neutral stimu- 
lus such as a light or tone is followed by an unconditioned stimulus (US) such as shock it soon becomes a conditioned stimulus (CS) capable of eliciting a conditioned response (CR) in the absence of the US. Fear conditioning procedures also illustrate how conditioning processes may go awry and lead to anxiety disorders (Mineka \& Oehlberg, 2008). For instance, conditioned suppression is a model of the inhibition of on-going learned (operant) behavior through presentations of a fear-eliciting CS (Estes \& Skinner, 1941). Conditioned suppression models of anxiety involve several interlinked phases. First, an operant response, such as bar pressing, is trained. Second, fear conditioning occurs during which a CS comes to elicit fear CRs. Finally, the test for conditioned suppression involves representing the CS during the operant task and recording any reduction in ongoing behavior in the presence of the CS and in its absence. Conditioned suppression has been extensively studied using a wide range of tasks with both nonhumans (e.g. Blackman \& Scruton, 1973; Estes \& Skinner, 1941) and humans (Arcediano, Ortega, \& Matute, 1996; Baeyens, Vansteenwegen, Hermans, Vervliet, \& Eelen, 2001; Baeyens et al., 2004; Havermans, Keuker, Lataster, \& Jansen, 2005; Nelson, Gregory, \& Sanjuan, 2012; Nelson \& Sanjuan, 2006).

Arcediano et al. (1996) developed a task called the "Martians" procedure (see also Franssen, Clarysse, Beckers, van Vooren, \& Baeyens, 2010) in which participants played a video game and learned to press the computer space bar to destroy invading Martians. The US was an intermittent white flashing of the screen (described in the context of the game as a defensive shield used by the Martians) with a change in the screen background color as the CS. Using a discrimination design, in which the US followed one CS (i.e., CS+) and not another (i.e., CS-), suppression ratios showed that participants suppressed responding in the presence of the CS+ but not the CS-. The findings of Arcediano et al. (1996) and subsequent extensions (Baeyens et al., 2001, 2004) are noteworthy for several reasons. First, an instructed US was used, which is a stimulus that lacks biological significance but which comes to significantly affect behavior by means of prior explicit verbal instruction (Baeyens et al., 2001). It remains to be seen whether a true model of human conditioned suppression can be devised that uses a biologically significant US. Second, operant responding during the US was in direct conflict with the task demands and was explicitly punished. That is, pressing the space bar during the instructed US would result in all of the invading Martians landing and the game would be lost. It is likely, therefore, that this feature influenced subsequent responding to the $\mathrm{CS}+$ and $\mathrm{CS}-$. The same rationale applies to other variants of the computer video game format used to assess conditioned suppression (e.g., Nelson \& Sanjuan, 2006).

VEs may provide a novel method of investigating conditioned suppression in a way that has clinical relevance for anxiety and applicability to real-world situations (Baas, Nugent, Lissek, Pine, \& Grillon, 2004). To date, VEs have shown considerable promise in both research and therapy for a number of psychological disorders (Cukor et al., 2009; Freeman, Pugh, Vorontsova, Antley, \& Slater, 2010). However, the majority of research has tended to use VEs to assess reactivity to conditioned stimuli, or to measure the effects of brief therapy on the disorder in question, rather than as an active part of the conditioning procedure. One notable exception (Grillon, Baas, Cornwell, \& Johnson, 2006) used VEs to study the effect of context conditioning on the expression of behavioral avoidance. Participants were exposed to a non-immersed display of three VEs (a bank, a casino and a restaurant) in which a light CS was presented and followed by either predictable or unpredictable brief shock, depending on the context. Their findings showed that reliable context conditioning occurred in the three VEs, as evidenced by eyeblink startle responses, ratings of anxiety, and behavioral avoidance. It is important to note, however, that Grillon et al.'s (2006) task did not allow participants to progress at their own pace through the three different contexts; instead, exposure to the VE was predetermined (i.e., participants merely viewed each context on a display).

\subsection{A custom modification of a video game}

The purpose of the present study was to expand on such work by developing a fully interactive first-person-perspective VE with which to investigate conditioned suppression. Our paradigm was presented as a simple first-person shooter (FPS) video game in which participants navigated through a series of rooms in search of gold bars, which were hidden within crates distributed throughout the rooms. Participants were able to destroy (and thus open) crates by shooting them with their gun. The more gold participants collected, the higher their score at the end of the game.

The paradigm was created using Source SDK, the engine underlying the popular FPS Half-Life 2, which has previously been used to generate VEs for psychology research (Kozlov \& Johansen, 2010). One difficulty involved with using the Source SDK is that it is primarily designed as a tool for creating new games or for developing modifications ("mods") to the existing game; it is not specialized psychology software. It can, therefore, be difficult to obtain the necessary data required for psychological studies from the game. For instance, in Kozlov and Johansen's (2010) experiment, the results for each participant were obtained by the experimenter back-tracking through the maze after the participants had completed the experiment, and noting in which areas the boxes had been destroyed; a time-consuming process (M.D. Kozlov, personal communication, 19th September, 2011). It would instead be desirable to customize the game to provide automatic recording of the occurrence of stimuli or responses made by the participants. Without this facility to accurately record response timing, much of the information essential for certain investigations (such as calculating response rates or intervals) would be lost. One of the main goals of the current study was therefore to assess the feasibility of implementing a data logging system that could automatically record all the necessary information as if the software were a specialized psychology tool. The experimenter can then simply retrieve a log file containing all the necessary information to analyze participants' behavior within the paradigm.

Nacke, Lindley, and Stellmach (2008) described the implementation of such a data logging system for Half-Life 2 . This system is highly sophisticated and covers a wide range of information that a researcher might wish to extract from a participant's performance within the game VE (such as when the player sustains an injury or interacts with another character). Here, we followed similar principles to create a simplified data logging system for our conditioned suppression paradigm. This was achieved by creating a customized logical entity in Visual C++ and then placing this entity within the game map (see Nacke et al. (2008), and the Valve Developer Community, https://www.developer.valvesoftware.com, for details). The entity was then configured to receive inputs from various other entities or events (such as a shot being fired, a crate being hit, or a light switching on) within the game level. By calling the appropriate function when the each specific input was received, the entity then logged the occurrence of that event and its time of occurrence. Using this method, we were able to record the precise timing of each CS+, CS-, US, and all responses performed by the participants. With these data, we were able to examine the effects of the various stimuli on our participants' behavior.

In nonhuman studies of conditioned suppression, the primary dependent measure is the response rate of the subject (e.g., rate of bar pressing). The advantage of using a customized VE paradigm is that multiple, subtle measures of behavior can be obtained. For 
example, basic operant responding is recorded as the number of shots fired using the weapon. However, our participants were required to aim at targets. Thus while some of the shots fired by participants hit the desired targets (crates), inevitably some shots were also off-target. We therefore can identify an additional dependent variable - the total number of shots that hit the desired targets, or "hits". In addition, since the overall goal was not just to hit the crates but to find gold by destroying those crates, and since destruction of a crate requires multiple hits, a third dependent variable, targets destroyed or "breaks" may also be identified. Finally, in most contemporary video games, there is a further statistic that is often very relevant; shot accuracy. In many games (and as in real life), ammunition for weapons is often in limited supply, and it is paramount to utilize available ammunition effectively; in other words, to make as many accurate shots as possible from the total number of shots fired. Here then, shot accuracy is calculable as a percentage of hits over shots.

The present paper reports preliminary findings from a study designed to investigate human conditioned suppression in a VE paradigm. We predicted suppression of shooting behavior in the presence of the CS+ but not in the presence of the CS-, for each of our dependent measures.

\section{Methods}

\subsection{Participants}

Thirty-two students and staff (19 female, 13 male, mean age 25 years) were recruited from Swansea University and participated for either $£ 6$ or partial course credit. The experiment was conducted in accordance with British Psychological Society ethical standards, and all participants provided written informed consent.

\subsection{Apparatus and materials}

A Dell Optiplex 755 PC running Microsoft Windows XP and fitted with a 27" Iiyama monitor controlled stimulus presentations and recorded all responses. A Logitech Rumblepad II wireless joypad controlled movement throughout the VE. Customized game sounds and the white noise US were delivered via Grado SR60 headphones.

The VE was designed using the Valve ${ }^{\circledR}$ Hammer Editor, part of the Source SDK. The base code was the SDK base 2007 and the game configuration was Half-Life 2. The source code was edited using Microsoft Visual C++ Express Edition. The Steam client was used to launch the game.

The VE consisted of 21 rooms divided into two primary regions: the orientation area and the experiment area. The orientation area served to familiarize participants with the VE, the control system and appropriate actions to perform prior to entering the experimental area. There were four rooms in the orientation area: three training rooms and one teleport room. The experiment area consisted of 17 rooms: an initial landing area, to which participants were transported from the orientation phase, and 16 interlocking rooms comprising the main setting, two of which formed Phase 1, 12 formed Phase 2, and a further two formed Phase 3. Participants were prevented from returning to earlier rooms/levels by the automatic closing and locking of doors.

\subsection{Design and stimuli}

Each room was illuminated by a single light source emanating from a centrally positioned light fitting. The CS+ and CS - consisted of this light source changing color to red and green (counterbalanced across participants), which were displayed for $5 \mathrm{~s}$, respec- tively. Colors were chosen on the basis of pilot testing and, as a manipulation check, participants were asked at the end of the experiment to identify the functions of the red and green colors, respectively.

The US consisted of a $2 \mathrm{~s}$ burst of white noise (e.g. Baltissen \& Boucsein, 1986; Peri, Ben-Shakhar, Orr, \& Shalev, 2000). Prior to the commencement of the experiment, participants were instructed to select a noise level that they found to be "unpleasant or irritating without being unbearably painful". All participants selected a level within the range of $65-75 \mathrm{~dB}$.

\subsection{Procedures}

Participants were tested individually in a small, darkened experimental lab. Following an initial familiarization exercise, the main experiment consisted of three phases: operant training, Pavlovian conditioning, and testing (see Fig. 1).

\subsubsection{Familiarization}

Participants were given instructions by the experimenter on how to use the joypad to move about the VE. In the first room, participants were told how to move around and how to open doors, and were then directed to a second room in which they collected a gun and received instructions on how to use it. In a third room, which contained four wooden crates placed upon tables, one of which contained a set of gold bars, participants practiced shooting and destroying the crates. On uncovering the gold, participants received an onscreen message stating, "You found some gold! +100 points!" When all four of the crates were destroyed, participants were informed they would be teleported to the main part of the experiment.

\subsubsection{Phase 1: Operant training}

Phase 1 began when participants entered the next room. Participants were instructed that their primary aim was to find gold bars hidden in crates and that for each set of gold bars they discovered they would be awarded 100 points. Inside the room, there were two shelves on the north (in front as they walked through the door) and east (on the right) walls, respectively, with each shelf holding 24 crates in a $6 \times 4$ (width $\times$ height) arrangement. An adjoining room contained a similar arrangement, except that the shelves were on the south and east sides (front and left from the player's perspective) while all other elements of the room (lighting, textures of floor, ceiling and walls) were identical. The door to this adjoining room was initially in the open position, emphasizing that the two adjoining rooms essentially formed a single playing area, constituting Phase 1 . There were a total of 96 crates available in this phase. Each crate required four hits (i.e., fixed ratio [FR] 4) to be destroyed. Gold bars were placed in a randomly determined 6 of the 24 crates on each wall.

The criterion to successfully complete Phase 1 involved destroying a minimum of 80 of the 96 crates within $8 \mathrm{~min}$ (i.e., $83.3 \%$ destruction rate). If the criterion was met, then the phase ended and participants were informed that they could proceed to the next phase. If participants failed to reach criterion, they were excluded from analysis.

\subsubsection{Phase 2: Pavlovian conditioning}

Phase 2 began when participants entered the next room and consisted of a total of 12 interlocking rooms. The rooms were identical to those in Phase 1, with the exceptions that no crates were placed on the shelves and that small arrows were placed above the doors indicating the direction in which to proceed. Participants were instructed that their only task was to progress through the rooms. While progressing through the rooms, the stimuli were presented. A delay-conditioning procedure was used with a $5 \mathrm{~s}$ delay 

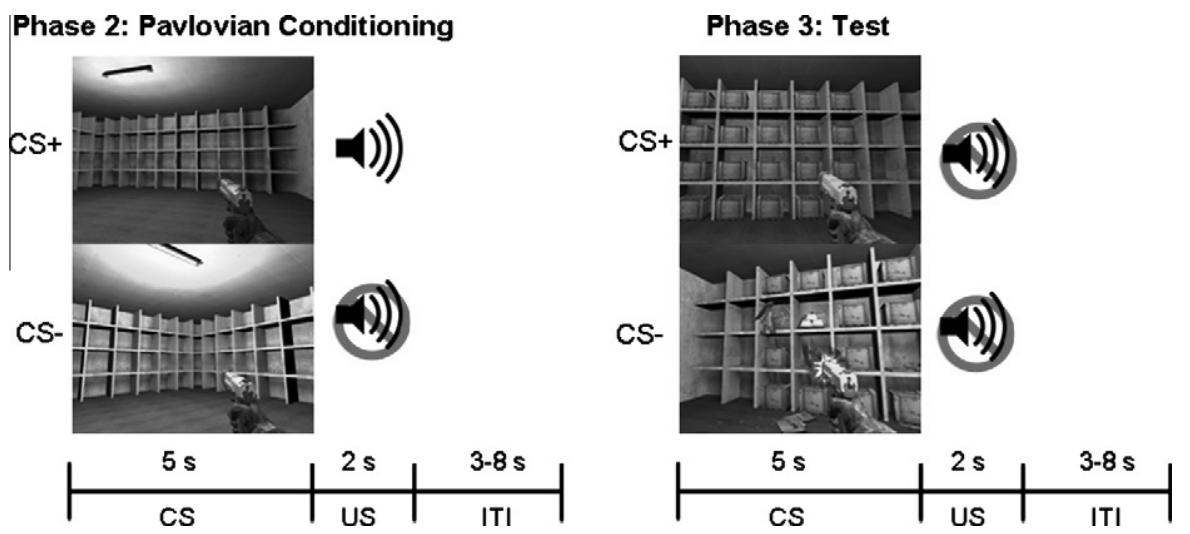

Fig. 1. Human conditioned suppression paradigm. Screen shots from Phase 2: Pavlovian conditioning showing the presentation of the CS+, which was followed (on $80 \%$ of trials) by the US, and the CS-, which was not followed by the US; and Phase 3: Test showing predicted behavior during unreinforced probe trials with responding (i.e., firing) suppressed during the presence of the CS+ but not during the presence of the CS-.

between $\mathrm{CS}+$ onset and US onset (i.e., the CS was $5 \mathrm{~s}$ in duration) and no trace interval separating CS+ and US (i.e., US onset co-occurred with CS+ termination). Participants were exposed to five presentations each of the CS+ and CS-, with the US following the CS+ on four of those five trials (i.e., CS + US contingency of 0.8 ). The US never followed the CS-. The CSs were arranged in a pseudorandom order across the first $150 \mathrm{~s}$ of Phase 2, with the constraint that no more than two CSs of the same type could occur consecutively, and the interval between the onset of one CS and the next was 10-15 s; thus with a CS duration of $5 \mathrm{~s}$ and US duration of $2 \mathrm{~s}$, the inter-trial interval (ITI) was between 3-8 $\mathrm{s}$ (as illustrated in Fig. 1). Participants were prevented from progressing to Phase 3 until they had experienced all ten CSs.

\subsubsection{Phase 3: Testing}

On completing Phase 2, participants entered the door to Phase 3 , where they were instructed that their task was identical to that of Phase 1. In all respects, Phase 3 was identical to Phase 1 except for the delivery of 10 probe trials, five each of the CS+ and CS-, again delivered in a pseudorandom order (as per Phase 2) across the first $150 \mathrm{~s}$ of the phase. There was no delivery of the US during this phase.

\subsection{Dependent measures and statistical analyses}

The dependent measures were; the total number of responses, (i.e., shots fired), total number of shots hitting desired targets (hits), total number of crates destroyed (breaks), and shot accuracy (percentage of total shots hitting targets) during Phase 3. For all measures, paired $t$-tests were used to directly compare relative frequencies during both CS+ and CS-. Suppression ratios (Annau \& Kamin, 1961) were then calculated to provide a normalized estimate of each measure against baseline ongoing behavior, and compared against the null value of 0.5 (indicating no suppression) using a one-sample $t$-test. Power analysis using $\mathrm{G} *$ Power 3 (Faul, Erdfelder, Lang, \& Buchner, 2007) with an $\alpha$-value of 0.05 , onetailed, assuming a medium effect size of 0.5 , indicated that the initial sample size $(n=32)$ was sufficient to produce a power of approximately 0.86 for these analyses.

\section{Results}

One participant failed to disclose red-green color blindness until after completing the experiment and was thus excluded from the analysis. Twenty-nine of the remaining 31 participants completed initial operant training at the first attempt and thus proceeded to Phases 2 and 3.

\subsection{Manipulation check and contingency awareness}

Participants gave the US a mean aversiveness rating of 3.93 on a 5 -point Likert scale (where 5 was the most aversive). In response to post-experimental questions regarding the extent to which they expected the US to follow either CS, participants gave a mean rating of 4.28 and 1.69 to the CS+ and CS-, respectively. This was a highly significant difference, $t(28)=10.316, p<0.0005$, indicating that participants were aware of the relationship between the CS+, CS - and US. Neither of these factors were correlated with the dependent measures.

\subsection{Direct comparison of dependent measures}

For each measure, we compared the totals obtained during the presence of the CS+ and the CS- respectively (see Table 1 ). It can be seen that the total overall responses (i.e., shots fired) were only marginally lower during the CS+ compared to the CS-. Analysis confirmed that the two mean response totals did not differ significantly, $t(28)=0.093, p=0.927$. A similar pattern was seen for the number of targets destroyed (breaks), $t(28)=-0.901, p=0.375$, but there was a marginally significant decrease in the number of shots on target, (i.e. hits) made during the CS+ compared to the CS-, $t(28)=-1.823, p=0.078$. Mean shot accuracy (accurate shots over total shots fired expressed as a percentage) was also significantly lower during the presence of the CS+ than that during the CS-, $t(28)=-2.248, p<0.05$.

\subsection{Suppression ratios}

As a more conventional means of assessing conditioned suppression in humans (e.g. Arcediano et al., 1996; Nelson \& Sanjuan, 2006), we also calculated suppression ratios as $A / A+B$, where $A$ is the total number of responses in the presence of the CS and $B$ is the total during an equivalent amount of time immediately preceding the CS. Suppression ratios of all dependent measures for both CSs are shown in Fig. 2. A suppression ratio of 0.5 indicates no suppres-

Table 1

Means (and standard deviations) for all dependent measures in the presence of the $\mathrm{CS}+$ and $\mathrm{CS}-$.

\begin{tabular}{lcc}
\hline & \multicolumn{1}{l}{ CS+ } & CS- \\
\hline Shots & $37.00(12.06)$ & $37.26(10.89)$ \\
Hits & $23.39(9.27)$ & $27.52(8.70)$ \\
Breaks & $5.87(2.38)$ & $6.57(2.38)$ \\
Accuracy (\%) & $65.96(16.41)$ & $74.07(14.18)$ \\
\hline
\end{tabular}




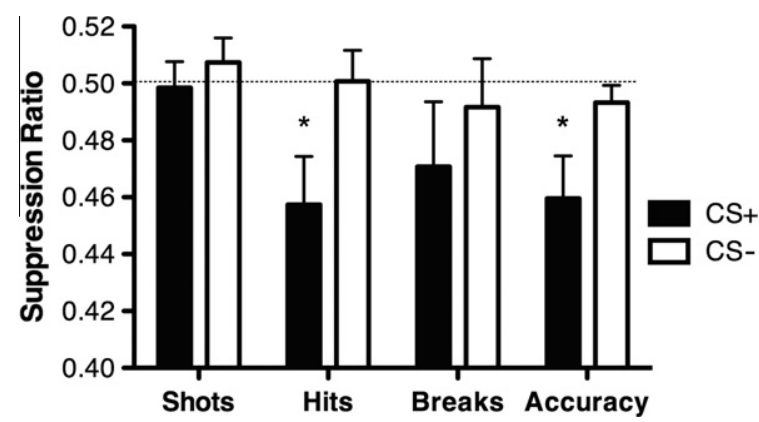

Fig. 2. Mean suppression ratio of total responses, hits (responses hitting valid targets), breaks (number of targets destroyed), and accuracy (hits over responses). The dashed horizontal line indicates no suppression. Error bars show standard errors. ${ }^{*} p<0.05$.

sion. As expected, during the CS-, none of the dependent measures were significantly suppressed (all $p s>0.2$ ). However during the CS+, while neither the suppression ratio for responses or breaks differed significantly from 0.5 (both $p s>0.2$ ), both hits, $t(28)=-2.514, p<0.05$, and accuracy, $t(28)=-2.701, p<0.05$, were significantly suppressed. This suggests that the presence of the CS+ impaired participants' ability to hit targets without affecting their overall rate of responding.

\section{Discussions}

Our findings reveal evidence of conditioned suppression of accurate responding (total hits and shot accuracy) elicited by an aversive CS in a first-person perspective VE task. As such, these findings extend the literature on conditioned suppression in humans (e.g., Arcediano et al., 1996; Franssen et al., 2010; Havermans et al., 2005) to a novel VE paradigm that includes multiple dependent measures. However, in contrast to our predictions, we did not obtain suppression of overall responses. We shall presently outline some potential explanations for this outcome. However, this finding may perhaps be considered to reflect a strength rather than a limitation of our paradigm, by indicating greater sensitivity in detecting subtle differences in ongoing operant behavior and illustrates the usefulness of VEs in capturing effects that would not manifest in a standard conditioned suppression paradigm.

Our results are broadly consistent with the literature on the adverse effects of anxiety on motor performance (Gucciardi \& Dimmock, 2008; Masters, 1992; Mullen \& Hardy, 2000). A potential explanation for our findings is that the fear-eliciting properties of the CS+ resulted in increased anxiety and a decrease in participants' ability to aim accurately at the targets. This interpretation would suggest that participants did indeed learn the relationship between the CS+ and the US (and between the CS - and the US), i.e. that the US was predicted by the CS+ (and not the CS-). Assuming that participants found the US sufficiently aversive (as the postexperimental ratings suggested), then the delivery of the CS+ would be expected to create an increased level of anxiety (compared to both baseline and to that during $\mathrm{CS}$ - presentation) and a concomitant decrease in ability to aim accurately.

A second explanation for the current findings can be presented in terms of response competition - that is, where execution of the operant response in question is prevented by the execution of an alternative, incompatible response. To explain further; while participants were powerless to affect or intercept the delivery of the US, they were not explicitly informed that this was the case. In other words, they were not aware that US delivery was beyond their control. During the presence of the CS+, participants may then have performed some other activity, besides shooting at targets, to try and prevent the delivery of the US. For instance, participants may have reasoned that if they could eliminate the CS+ then the US would not be delivered. Therefore, participants may have attempted to destroy the light-source by shooting at it when it changed to the undesirable color. While anecdotal reports indicated that this was the case for at least two participants in our sample, it is difficult to evaluate whether response competition prompted participants to seek to prevent the occurrence of the US because the VE task did not record such unpredicted shooting responses. Future research should consider this possibility by logging all such instances of targeted shooting.

Perhaps the most interesting aspect of the present study is that we induced suppression of shot accuracy without producing overall conditioned suppression of operant responding rates. Our manipulation checks demonstrate that participants recognized the relationship between the CS+ and the US and that a high rating was given to the aversiveness of the US. As suggested earlier, while participants may have experienced anticipatory anxiety (Cain, Dunsmoor, LaBar, \& Mitroff, 2011) the task instructions may have specified that freezing behavior was an incompatible response. An implication of this finding is that suppression of operant behavior may not always be a true reflection of experiential anxiety; or rather that anxiety may not always be reflected in suppression of ongoing behavior. To adequately assess the effects that cue-elicited anxiety has on behavior, it may therefore be necessary to appeal to a range of more complex behaviors, such as the dependent measures employed in the current study, rather than reply upon than simple response-outcome schedules (Bond, Blackman, \& Scruton, 1973; Kamin, 1961) as measures. In a paradigm such as the current one, measures of accuracy are clearly a more important reflection of conditioning, and of anxiety, than overall responding.

The present findings have implications for police, military and other security personnel who often have to perform complicated motor skills such as aiming at targets under pressure. Indeed, a recent study by Nieuwenhuys and Oudejans (2010) has obtained similar effects in a real-life police training exercise. Seven police officers participated in this shooting exercise, which consisted of one condition in which there was an opponent returning fire, thus creating a high anxiety condition, and the low-anxiety condition in which the opponent was non-threatening. Nieuwenhuys and Oudejans (2010) found significantly poorer performance in the condition with the opponent returning fire. This supports the main assumptions of the literature suggesting that human behavior in VEs tends to mirror human behavior in real environments and that VEs are an appropriate tool both for the investigation of human behavior, for developing behavioral therapies, learning tools, and for simulating reality (Baumann \& Sayette, 2006; Difede \& Hoffman, 2002). Conducting training exercises using VEs, for instance, offers several advantages such as greater control over the phenomena of interest, increased safety and reduced cost.

We must, however, emphasize caution in the interpretation of our results. Firstly, although we did obtain statistically significant suppression of accurate shots (in both absolute terms and as a proportion of total shots), when directly comparing total hits in the presence of the CS+ and CS-, the effect was marginal. Only when accurate responses were considered as a percentage of total responses was accuracy significantly lower during the CS+. This decline in accuracy therefore reflects not just a lower proportion of targets hit but also an increase in the number of errant shots, a subtle but nevertheless important point. Future research should seek to develop versions of the present procedures that clearly identify the nature of inaccurate responses; that is, whether they are simply "misses" or the result of aiming at other entities that could be considered potential targets. Secondly, our sample size, although sufficient (Faul et al., 2007), might still be considered small, and hence future research should consider using larger samples to confirm the robustness of the suppressive effects seen in 
the current study. Thirdly, since the paradigm is novel, its effectiveness is not established, compared to, for example, the standard free-operant procedure (Ferster, 1953). Further refinements to the paradigm may result in a more effective method for assessing conditioned suppression. Finally, the use of the present VE paradigm to evaluate conditioned suppression confers many advantages, but attention must also be drawn to the limitations involved. Participants in our study were free to explore and behave as they wished within the VE, and while this enhances ecological validity, it may also generate unintended difficulties. For instance, participants may not always adhere to the anticipated course of action and thus there is no guarantee that they will maintain a constant, rapid rate of shooting because, at any given moment, a participant could abandon shooting, navigate elsewhere in the VE, or simply take a break. If behaviors such as these occurred during CS presentations, then our measures of suppression would be inadvertently affected. These are, however, some of the risks inherent in using a free-operant VE task to conduct ecologically valid research on conditioning processes involved in anxiety.

Further research should seek to develop customized modifications of the VE task that permit greater adherence to the experimental predictions, such as, a task involving simple rule learning, responses to punishment, or where the participant has a degree of control over the occurrence of the US. If, for example, the CS produces the US while participants are shooting, but does not produce it during navigation (i.e., while participants are not shooting), will suppression of shooting then be observed when the CS is subsequently presented? Arcediano et al.'s (1996) findings suggest that greater reduction in operant responding may be obtained if responding during the US (or CS) is explicitly punished by an enhancement of the US. Other aversive USs, such as electric shock, or different CS modalities (e.g., tones instead of lights) should be studied to assess the extent to which the present findings generalize to other situations. Additionally, the current paradigm could potentially be adapted as a training tool, perhaps to improve response accuracy under conditions promoting anxiety, or to learn to ignore distracting stimuli. VEs have previously been utilized to facilitate performance in a range of cognitively demanding settings; for instance, Dye, Green, and Bavelier (2009) demonstrated that a video game could be used to increase the speed of action processing (see also Achtman, Green, \& Bavelier, 2008).

\subsection{Conclusion}

We devised a novel VE paradigm to investigate conditioned suppression in humans. We found that an aversive conditioned stimulus significantly impaired accurate responding while not suppressing operant responding per se. This outcome has important implications for understanding the effects of anxiety on complex behavior, and is relevant to the training of any profession involving the use of firearms. Furthermore, the current findings highlight the potential for using VEs to uncover subtle behavioral manifestations of anxiety (and potentially other psychological processes) that traditional paradigms might not so easily reveal. Finally, the concordance between these findings and those obtained in real-life exercises adds further credence to the notion that behavior in VEs mirrors that in natural environments, and enhances the validity of VEs as a tool in research and therapy.

\section{Acknowledgments}

We thank Michail Kozlov, Mark Johansen, and Sophie Stellmach for providing additional details of their respective work.

\section{References}

Achtman, R. L., Green, C. S., \& Bavelier, D. (2008). Video games as a tool to train visual skills. Restorative Neurology and Neuroscience, Special Issue on Visual System Damage and Plasticity, 26(4-5), 435-446.

Annau, Z., \& Kamin, L. J. (1961). The conditioned emotional response as a function of intensity of the US. The Journal of Comparative and Physiological Psychology, 54(4), 428-432.

Arcediano, F., Ortega, N., \& Matute, H. (1996). A behavioural preparation for the study of human Pavlovian conditioning. Quarterly Journal of Experimental Psychology, 49B(3), 270-283.

Baas, J. M., Nugent, M., Lissek, S., Pine, D. S., \& Grillon, C. (2004). Fear conditioning in virtual reality contexts: A new tool for the study of anxiety. Biological Psychiatry, 55(11), 1056-1060.

Baeyens, F., Vansteenwegen, D., Hermans, D., Vervliet, B., \& Eelen, P. (2001). Sequential and simultaneous feature positive discriminations: Occasion setting and configural learning in human Pavlovian conditioning. Journal of Experimental Psychology: Animal Behavior Processes, 27(3), 279-295.

Baeyens, F., Vervliet, B., Vansteenwegen, D., Beckers, T., Hermans, D., \& Eelen, P. (2004). Simultaneous and sequential feature negative discriminations: Elemental learning and occasion setting in human Pavlovian conditioning. Learning and Motivation, 35(2), 136-166.

Baltissen, R., \& Boucsein, W. (1986). Effects of a warning signal on reactions to aversive white noise stimulation: Does warning 'short-circuit' habituation? Psychophysiology, 23(2), 224-231.

Baumann, S. B., \& Sayette, M. A. (2006). Smoking cues in a virtual world provoke craving in cigarette smokers. Psychology of Addictive Behaviors, 20(4), 484-489.

Beckers, T., Krypotos, A.-M., Boddez, Y., Effting, M., \& Kindt, M. (in press). What's wrong with fear conditioning? Biological Psychology.

Blackman, D. E., \& Scruton, P. (1973). Conditioned suppression and discriminative control of behavior. Learning and Behavior, 1(2), 90-92.

Blascovich, J., Loomis, J., Beall, A. C., Swinth, K. R., Hoyt, C. L., \& Bailenson, J. N. (2002). Immersive virtual environment technology as a methodological tool for social psychology. Psychological Inquiry, 13(2), 103-124.

Bond, N. W., Blackman, D. E., \& Scruton, P. (1973). Suppression of operant behavior and schedule induced licking in rats. Journal of the Experimental Analysis of Behavior, 20(3), 375-383.

Cain, M. S., Dunsmoor, J. E., LaBar, K. S., \& Mitroff, S. R. (2011). Anticipatory anxiety hinders detection of a second target in dual target search. Psychological Science, 22(7), 866-871.

Cukor, J., Spitalnick, J., Difede, J., Rizzo, A., Rothbaum \& Barbara, O. (2009). Emerging treatments for PTSD. Clinical Psychology Review, 29(8), 715-726.

Di Giusto, J. A., Di Giusto, E. L., \& King, M. G. (1974). Heart rate and muscle tension correlates of conditioned suppression in humans. Journal of Experimental Psychology, 103(3), 515-521.

Difede, J., \& Hoffman, H. G. (2002). Virtual reality exposure therapy for World Trade Center post-traumatic stress disorder: A case report. CyberPsychology $\mathcal{E}$ Behavior, 5(6), 529-535.

Dye, M. W. G., Green, C. S., \& Bavelier, D. (2009). Increasing speed of processing with action video games. Current Directions in Psychological Science, 18(6), 321-326.

Estes, W. K., \& Skinner, B. F. (1941). Some quantitative properties of anxiety. Journal of Experimental Psychology, 29, 390-400.

Faul, F., Erdfelder, E., Lang, A.-G., \& Buchner, A. (2007). G*power 3: Aflexible statistical power analysis program for the social, behavioral, and biomedical sciences. Behavior Research Methods, 39(2), 175-191.

Ferster, C. B. (1953). The use of the free operant in the analysis of behavior. Psychological Bulletin, 50(4), 263-274.

Franssen, M., Clarysse, J., Beckers, T., van Vooren, P. R., \& Baeyens, F. (2010). A free software package for a human online conditioned suppression preparation. Behavior Research Methods, 42(1), 311-317.

Freeman, D., Pugh, K., Vorontsova, N., Antley, A., \& Slater, M. (2010). Testing the continuum of delusional beliefs. Journal of Abnormal Psychology, 119(1), 83-92.

Grillon, C., Baas, J. M. P., Cornwell, B., \& Johnson, L. (2006). Context conditioning and behavioral avoidance in a virtual reality environment: Effect of predictability. Biological Psychiatry, 60(7), 752-759.

Gucciardi, D. F., \& Dimmock, J. A. (2008). Choking under pressure in sensorimotor skills: Conscious processing or depleted attentional resources? Psychology of Sport and Exercise, 9(1), 45-59.

Guitton, M. J. (2012). Living in the Hutt space. Immersive process in the Star Wars role-play community of second life. Computers in Human Behavior, 28(5), 1681-1691.

Havermans, R. C., Keuker, J., Lataster, T., \& Jansen, A. (2005). Contextual control of extinguished conditioned performance in humans. Learning and Motivation, 36(1), 1-19.

Huff, N. C., Zielinski, D. J., Fecteau, M. E., Brady, R., \& LaBar, K. S. (2010). Human fear conditioning conducted in full immersion 3-dimensional virtual reality. Journal of Visualized Experiments, 42, e1993.

Kamin, L. J. (1961). Trace conditioning of the conditioned emotional response. Journal of Comparative and Physiological Psychology, 54(2), 149-153.

Kozlov, M. D., \& Johansen, M. K. (2010). Real behavior in virtual environments: Psychology experiments in a simple virtual-reality paradigm using video games. Cyberpsychology, Behavior, and Social Networking, 13(6), 711-714. 
Lomanowska, A. M., \& Guitton, M. J. (2012). Spatial proximity to others determines how humans inhabit virtual worlds. Computers in Human Behavior, 28(2), 318-323.

Masters, R. S. W. (1992). Knowledge, knerves and know-how: The role of explicit versus implicit knowledge in the breakdown of a complex motor skill under pressure. British Journal of Psychology, 83(3), 343-358.

Mineka, S., \& Oehlberg, K. (2008). The relevance of recent developments in classica conditioning to understanding the etiology and maintenance of anxiety disorders. Acta Psychologica, 127(3), 567-580.

Mullen, R., \& Hardy, L. (2000). State anxiety and motor performance. Testing the conscious processing hypothesis. Journal of Sports Sciences, 18(10), 785-799.

Nacke, L., Lindley, C., \& Stellmach, S. (2008). Log who's playing: Psychophysiological game analysis made easy through event logging. In P. Markopoulos, B. deRuyter W. A. IJsselsteijn, \& D. Rowland (Eds.). Fun and Games (Vols. 150-157). Berlin: Springer-Verlag.
Nelson, J. B., Gregory, P., \& Sanjuan, M. d. C. (2012). The extinction context enables extinction performance after a change in context. Behavioural Processes, 90(3), 372-377.

Nelson, J. B., \& Sanjuan, M. d. C. (2006). A context-specific latent inhibition effect in a human conditioned suppression task. Quarterly Journal of Experimental Psychology, 59(6), 1003-1020.

Nieuwenhuys, A., \& Oudejans, R. R. D. (2010). Effects of anxiety on handgun shooting behavior of police officers: A pilot study. Anxiety, Stress \& Coping, 23(2), 225-233.

Peri, T., Ben-Shakhar, G., Orr, S. P., \& Shalev, A. Y. (2000). Psychophysiologic assessment of aversive conditioning in posttraumatic stress disorder. Biological Psychiatry, 47(6), 512-519.

Slater, M., Antley, A., Davison, A., Swapp, D., Guger, C., Barker, C., et al. (2006). A virtual reprise of the Stanley Milgram obedience experiments. PLoS ONE, 1, e39. 